\title{
Persistent Asymptomatic Plasmodium falciparum Driven Anemia in a Treatment Re-infection Pediatric Cohort in a Rural Health District of Cameroon
}

Ouambo HF ${ }^{1,2}, \mathrm{Ngu} \mathrm{LN}^{2,3}$, Lissom $\mathrm{A}^{2,4}$, Ngoh AA ${ }^{2,5}$, Okoli $\mathrm{AS}^{6}$, Atabonkeng PE $\mathrm{PE}^{7}$, Takoua $\mathrm{D}^{8}$, Garcia $\mathrm{R}^{9}$, Gutiérrez $\mathrm{A}^{9}, \mathrm{Chukwuma} \mathrm{GO}^{2,10}, \mathrm{Ngouadjeu}^{1}$ $\mathrm{E}^{11}$, Okeke MI ${ }^{12}$, Esimone $\mathrm{CO}^{13}$, Achidi EA ${ }^{1}$, Mbacham WN ${ }^{2,14,15}$, Kaptue L $^{16}$, Park CG $^{17,18}$, Waffo AB $^{19,20}$, Assob ANC ${ }^{1}$ and Nchinda GW ${ }^{2 *}$

${ }^{1}$ Department of Medical Laboratory Sciences, University of Buea, Cameroon

${ }^{2}$ Laboratory of Vaccinology/Biobanking, CIRCB, Cameroon

${ }^{3}$ Department of Biochemistry, University of Yaoundé I, Cameroon

${ }^{4}$ Department of Animal Biology and Physiology, University of Yaoundé I, Cameroon

${ }^{5}$ Department of Biomedical Sciences, University of Dschang, Cameroon

${ }^{6}$ Gen $\varnothing k$, Centre for Biosafety, Tromsø, Norway

${ }^{7}$ Department of Biochemistry, University of Dschang, Cameroon

${ }^{8}$ Department of Biomedical Laboratory Sciences, University of Ngaoundéré, Cameroon

${ }^{9}$ Centre de Santé Catholique de Bikop, Cameroon

${ }^{10}$ Department of Medical Lab Sciences; Nnamdi Azikiwe University, Awka, Nigeria

${ }^{11}$ Faculty of Medicine and Pharmaceutical Sciences, University of Douala, Cameroon

${ }^{12}$ Department of Medical Biology, Faculty of Health Sciences, Molecular Inflammation Research Group, UiT The Arctic University of Norway, N-9037 Tromsø, Norway

${ }^{13}$ Department of Pharmaceutical Microbiology \& Biotechnology, Nnamdi Azikiwe University Awka, Nigeria

${ }^{14}$ The Biotechnology Center, University of Yaoundé I, Cameroon

${ }^{15}$ The Department of Biochemistry and Physiology, Faculty of Medicine, University of Yaoundé I, Cameroon

${ }^{16}$ Université des Montagnes, Bangangté, Cameroon

${ }^{17}$ Laboratory of Immunology, Brain Korea 21 PLUS Project for Medical Science, Severance Biomedical Science Institute, Yonsei University College of Medicine, Seoul 03722, Republic of Korea

${ }^{18}$ Laboratory of Cellular Physiology and Immunology and Chris Browne Center for Immunology and Immune Diseases, Rockefeller University, New York, NY 10065, USA

${ }^{19}$ Department of Biological Sciences/College STEM 1627 Hall Street Montgomery, AL 36101, USA

${ }^{20}$ Center for Nano Biotechnology Research, 1627 Harris Way Montgomery, AL 36104, USA

\begin{abstract}
Background: Asymptomatic Plasmodium falciparum infection (APFI) resulting from partial immunity is beneficial to individuals living in malaria hyper endemic regions like Cameroon. However APFI sustains not only persistent malaria transmission in the general population but equally accounts for complications related to untreated malaria such as anemia. The aim of this study therefore was to determine the rate of anemia within a population of children with persistent APFI, living in a rural area of Cameroon.
\end{abstract}

Materials and methods: A cohort of children from nursery and primary school was monitored for four months in the Bikop Health District of Cameroon. Using pretested questionnaire data was collected from 80 children less than 13 years old during the months of November 2016 and February 2017. Blood samples were collected twice by venipuncture during this period. Children with acute symptoms of malaria, fever or chills and children recently sick or under malaria treatment were excluded. Using malaria rapid diagnostic kits (RDTs) and fluorescent microscopy we screened for malaria positive people and also for the hemoglobin level using a hemoglobinometer.

Results: The median age of participants was 6.5 (4-10). The rate of APFI increased from $41.25 \%$ in November 2016 to $65 \%$ in February 2017 irrespective of the use of Long Lasting Insecticidal Nets (LLIN). Similarly, the rate of anemia also increased $(100 \%$ for the $2-5,100 \%$ for the $6-9$ years and $92.31 \%$ for the $10-13$ years old children respectively). The changes in asymptomatic malaria associated anemia correlated positively $(r=0.93, p=0.002)$ with increasing Plasmodium falciparum parasitemia especially with respect to moderate anemia $(38.75 \%$ in the month of November and $50.00 \%$ in the month of February).

Conclusion: Thus asymptomatic Plasmodium falciparum parasitemia could be a driving force behind persistent anemia in school age children in rural areas of Cameroon.

Keywords: Asymptomatic malaria; Anemia; Parasitemia; Children; Plasmodium falciparum

List of Abbreviation: AL: Artemether Lumefantrime; APFI: Asymptomatic Plasmodium falciparum Infection; CIRCB: Centre International de Reference Chantal Biya; EDTA: Ethylene Diamine Tetra Acetate; HiCN: Hemoglobin Cyanide; HRP 2: Histidin Rich Protein; LDH: Lactate Deshydrogenase; LLIN: Long Lasting Insecticidal Net; P. falciparum: Plasmodium falciparum; RDT: Rapid Diagnostic Test

\section{Introduction}

Malaria is the deadliest disease in the human story and it still infects hundreds of millions of people every year. Despite being preventable
*Corresponding author: Nchinda GW, Laboratory of Vaccinology/Biobanking, CIRCB, Cameroon, Tel: 237 676523909; Fax: 237222315456; E-mail: nsehleseh@gmail.com

Received June 20, 2019; Accepted June 31, 2019; Published July 07, 2019

Citation: Ouambo HF, Ngu LN, Lissom A, Ngoh AA, Okoli AS, et al. (2019) Persistent Asymptomatic Plasmodium falciparum Driven Anemia in a Treatment Re-infection Pediatric Cohort in a Rural Health District of Cameroon. J Blood Lymph 9: 248.

Copyright: (c) 2019 Ouambo HF, et al. This is an open-access article distributed under the terms of the Creative Commons Attribution License, which permits unrestricted use, distribution, and reproduction in any medium, provided the original author and source are credited. 
and treatable, malaria is a leading cause of death and disease across many tropical and subtropical countries. In fact, in 2016, there were an estimated 216 million cases of malaria in 91 countries, with 445000 deaths. About $90 \%$ of all malaria deaths occurred in Africa and the most afflicted were children under 5 years old [1]. Most children experience their first malaria infections during the first or second year of life, when they have not yet acquired adequate immunity, increasing their vulnerability. In addition, repeated malaria infections contribute significantly to mortality due to severe anemia, and by increasing their susceptibility to other common childhood illnesses, such as diarrhea and respiratory tract infections [2,3]. In Addition to this, late presentation to the hospital, inadequate management and unavailability of effective drugs contribute significantly to the malaria morbidity and mortality [1].

Malaria is caused by the protozoan parasite Plasmodium, and six plasmodial species present a significant health threat for humans: among which $P$. falciparum and $P$. vivax the most important in terms of deaths and in a lesser extent $P$. ovale curtisi, $P$. ovale wallikeri, and P. malariae. P. knowlesi who have recently emerged as a local but important causes of the disease. Although zoonotic in their aetiology, there is no definite evidence of primary human-to-human transmission yet $[1,2,4]$. Malaria is transmitted by the anopheles mosquitoes; in fact, the female anopheles mosquito infects the host during a blood meal by injecting the parasites into the host dermis and thereby, the life cycle progress into the human organism [5].

In Cameroon, malaria continue to be a major public health problem despite all the efforts made by the National Malaria Control Programme [6]. The estimated mortality rate of malaria is $116 / 1000$, and it is higher than the bordering countries (104/1000) [7]. The rate of the malaria transmission depends on the region of Cameroon; in fact, except the northern regions, the transmission is perennial in most of the other regions [7]. Most data concern clinical cases, with almost 900,000 clinical cases occurring every year, whereas no data has been published on subclinical cases in Cameroon [6,7]. Indeed, APFI refers to the presence of Plasmodium falciparum in the blood without symptoms. Carriers become a reservoir for the parasite and because they do not seek treatment, they are largely responsible for persistent transmission of the disease and thereby lead to complications of malaria $[3,8]$. Although the suspicion of malaria has always been based on acute symptoms of the disease like fever and chills, it is currently known that these acute symptoms occur in partially immune or non-immune individuals especially children in high transmission settings and visitors to endemic areas [3]. Thus, APFI results from partial immunity which controls but does not completely eliminate the infection $[3,8]$.

Therefore, there is a need to consider the burden of APFI in the establishment of the malaria policies, particularly in areas with high transmission rate. We carried out this study in the area of Bikop, situated in the heart of the rainforest in the Centre Region of Cameroon, an area with high malaria endemicity and perennial transmission [7]. Children living in this area are thereby highly exposed and this could enable their immune system to develop adequate immune response early in their live. However this situation could cause the appearance of APFI cases and associated anemia among the youngest children. For this purpose we ascertained the rate of asymptomatic Plasmodium falciparum infection and associated anemia within the population of children living in Bikop health area of Cameroon.

\section{Materials and Methods}

\section{Description of the study area}

This study was carried out in Bikop $\left(3^{\circ} 31^{\prime} 00.0^{\prime \prime} \mathrm{N} 11^{\circ} 25^{\prime} 00.0^{\prime \prime} \mathrm{E}\right)$
[9], a health district located in the heart of the rainforest, in the Center Region of Cameroon. This is $48 \mathrm{Km}$ away from Yaounde the City Capital of Cameroon. The Bikop health district covers 28 rural communities with an estimated population of 30,000 inhabitants [10]. The climate is typically equatorial with an average annual temperature of $23.5^{\circ} \mathrm{C}$ and mean annual rainfall of $831.7 \mathrm{~mm}[9,10]$. There are two seasons; the dry season from November to February and June to August; the wet season from February to May and September to November. Agriculture and fishing are the main sources of livelihood $[9,10]$.

\section{Study design and ethical considerations}

A cohort study was carried out for four months from November 2016 to February 2017. Ethical clearance was obtained from the Cameroon National Ethics Committee (N² 2018/01/969/CE/CNERSH/ SP), Meetings were organized with the parents, the school staff and health facility staff, during which the project objectives, methods and possible benefits/risks were clearly explained. Thereafter, pupils of the nursery and primary school of Bikop were invited to participate in the survey. Assented participants were interviewed using a pretested questionnaire, to collect socio-demographic information and history of previous episodes of malaria, and treatment regiments. Parents or guidants were interviewed for participants under 5 years old. Each child received a general clinical evaluation from a clinicians of the Bikop Catholic health facility. Children with acute symptoms of malaria, fever $\left(>37.5^{\circ} \mathrm{C}\right)$ or chills, and children recently sick [three months prior to data collection] or under antimalarial or anemia treatment were excluded from the study. All diagnosed cases of malaria and anemia were treated free of charge using artesunate-lumefantrine combination therapy for malaria, and ferrous iron-folic acid for anemia.

\section{Samples collection and processing}

During the month of November 2016, a total of 123 children were screened for the study. Temperature was recorded using an infrared body thermometer (Shenzhen calibeur industries, China). During the study period $5 \mathrm{~mL}$ of Blood were collected using sterile disposable syringes into labelled ethylene diamine tetra acetate (EDTA) tubes, at the Bikop Catholic health facility. While the Rapid Diagnostic Test (SD Bioline Malaria Ag Pf/Pan ${ }^{\mathrm{nt}}$ ) for malaria and the hemoglobin concentration (hemoglobinometer Urit-12) test was immediately on site, the remaining blood were transported in an ice cooler box to CIRCB for fluorescent microscopy and sample processing for storage. Based on the selection criteria, only 80 children were finally retained in the study during the sampling period of February 2017 thereby constituting the cohort of interest in our study.

\section{Laboratory Investigations}

\section{Malaria diagnostic}

The SD Bioline Malaria Ag Pf/Pan ${ }^{\text {Tx }}$ (country) RDT uses Histidine-Rich Protein 2 (HRP 2) to detect P. falciparum and Lactose Dehydrogenase (LDH) for Plasmodium vivax, Plasmodium ovale and Plasmodium malariae [11-13]. This test is a WHO prequalified test useful for the region where all malaria species are circulated, and it has a sensitivity of $99.7 \%$ for P. falciparum and $95.5 \%$ for P. vivax, with a specificity of $99.3 \%[11,12]$. The tests were used according to manufacturer's instructions [11]. To ensure the validity of the results, RDTs were read within a timeframe of maximal 15 minutes by two independent laboratory technicians $[11,12]$.

A fluorescent microscopy based malaria rapid diagnostic test from Partec [Partec GmbH, Germany] was also used for diagnosis of malaria 
Citation: Ouambo HF, Ngu LN, Lissom A, Ngoh AA, Okoli AS, et al. (2019) Persistent Asymptomatic Plasmodium falciparum Driven Anemia in a Treatment Re-infection Pediatric Cohort in a Rural Health District of Cameroon. J Blood Lymph 9: 248.

in CIRCB. Briefly $10 \mu \mathrm{l}$ of EDTA blood was added to ready-to-use prepared test slides incubated briefly according to the manufacturer's instruction and read with an $\mathrm{EVOS}^{\oplus}$ cell imaging fluorescent microscope (Thermoscientific, USA) The presence of distinct bright, shiny, tiny dots observed under the UV light indicated the presence of malaria parasites [14-16]. This was counted to determine the parisitaemia for each participant.

\section{Hemoglobin concentration determination}

Hemoglobin concentration was determine using a portable ahemoglobinometer Urit-12 (Urit medical electronic, Jiangsu, China) a fast hemoglobin and haematocrit test providing results within 10 seconds. Briefly $10 \mathrm{ul}$ of samples are dispensed into disposable microcuttes and read with the Urit-12. The instrument is factory precalibrated using $\mathrm{HiCN}$ (hemoglobin cyanide) standard, and absorbance of the test solution is automatically converted to concentration of Hemoglobin. The result is displayed in less than a minute.

\section{Data analysis}

Data were entered using excel 2013 and analyzed with the prism graph pad 6.0 statistical software. The associations between APFI and anemia were determined using Pearson's rank correlations. Group means were compared using analysis of variance (ANOVA) and Student's test.

\section{Results}

\section{Study population characteristics}

A total of 80 children were recruited from the 123 eligible participants screened. The eligible participants were excluded because they developed clinical malaria during the period of observation. Participants' age ranged from 2 to 13 years old with a median age of 4 (IQR: 3-4.75). The participants were categorize into three groups according to age. This consisted of group I (2-5), group II (6-9) and group III (10-13) years old respectively. Children under five years of age (group I) were the most represented subpopulation (Table 1). The proportion of boys and girls were approximately the same with $46.25 \%$ being girls and $53.75 \%$ boys. The mean body temperature was $36.26^{\circ} \mathrm{C}$ \pm 0.52 . The use of Long Lasting Insecticidal Net (LLIN) was limited as just $67.50 \%$ of all participants confirmed using them.

\section{Asymptomatic Plasmodium falciparum infection profile}

The rate of APFI increased from November 2016 to February 2017 irrespective of the age (Table 2). The increased rate was highest among children aged between 6 to 9 years (Table 2). This rate of APFI also increased within this period independently of the used of the LLIN (Table 2).

We also observed that, they was a positive correlation between the age and the increased parasitemia during the month of February $(\mathrm{r}=0.3144, \mathrm{p}=0.0045$ (Figure 1).

\section{Hemoglobin and anemia profiles of APFI children}

The mean level of hemoglobin decreased significantly from November 2016 to February 2017, in particular among children under five and children aged 10 to 13 years ( $2-5$ years: $12.56 \pm 2.13$ to $10.72 \pm$ 1.45 ; $10-13$ years: $11.95 \pm 1.08$ to $10.31 \pm 1.37 . \mathrm{p}<0.0001$ ) (Table 3 ). Most of the participants are suffering from moderate anemia independently of the age (Table 3 ).

Moreover, while the rate of APFI relapse cases increases, the rate of associated anemia also increases (Table 4);

The increased rate of APFI correlated positively with increasing anemia $(r=0.93, p=0.002$; (Table 5). Moreover, the increased parasitemia correlated with decreased hemoglobin level (Figure 2). This correlation significantly increased over the time (November 2016: $\mathrm{r}=0.5615, \mathrm{p}<0.0001$; February 2017: $\mathrm{r}=0.7430, \mathrm{p}<0.0001)$.

\section{Discussion}

These studies show that a significantly high number of school children in a remote area of Cameroon were asymptomatic carriers of Plasmodium falciparum parasites. Such high rate of asymptomatic Plasmodium falciparum infection (APFI) correlated positively with an increasing anemia especially moderate anemia. APFI represents an important threat for children health in rural areas because it sustains malaria transmission year round yea round thereby exacerbating morbidity in school age children. Nevertheless it could also be beneficial as it helps to maintain partially acquired immunity to malaria, thereby reducing the risk of severe disease [17].

The rate of APFI obtained in this study was higher with relative to the $6.8 \%$ reported in Ethiopia [18] and the 6.3\% in the Lao People's Democratic Republic [19]. This difference may be related to variations in the global malaria endemicity which is also directly linked to the transmission intensity [1,3]. For example in 2016 well over $71 \%$ of people living in Cameroon were highly exposed to malaria $[20,21]$ In contrast only $27 \%$ of people living in Ethiopia [20-22,] and $52 \%$ of those living in the Lao People's Democratic Republic [20,21-

\begin{tabular}{|c|c|c|c|c|}
\hline \multirow{2}{*}{\multicolumn{2}{|c|}{ Groups }} & $\mathbf{I}$ & II & III \\
\hline & & 2 to 5 years $(n=36)$ & 6 to 9 years $(n=21)$ & 10 to 13 years $(n=23)$ \\
\hline \multicolumn{2}{|c|}{ Age ranges (years): Median (IQR) } & $4(3-4.75)$ & $8(7.5-9)$ & $11(10-11)$ \\
\hline \multirow[t]{2}{*}{ Sex: $\mathrm{n}[\%]^{\mathrm{c}}$} & Female & $14(38.89)$ & $15(71.43)$ & $8(34.78)$ \\
\hline & Male & $22(61.11)$ & $6(28.57)$ & $15(65.22)$ \\
\hline \multicolumn{2}{|c|}{ Temperature $\left({ }^{\circ} \mathrm{C}\right)^{\mathrm{d}}:$ mean $\pm \mathrm{SD}^{\mathrm{e}}$} & $36.42 \pm 0.5$ & $36.24 \pm 0.44$ & $36.04 \pm 0.56$ \\
\hline \multicolumn{2}{|c|}{ LLIN*=yes: n (\%) } & $26(72.22)$ & $13(61.90)$ & $15(65.22)$ \\
\hline
\end{tabular}

*Long Lasting Insecticidal Net; aNumber; 'Inter Quartile Ranges; 'Percentage; ' ${ }^{\mathrm{d}}$ Body temperature; eStandard Deviation.

Table 1: Study population characteristics $(n=80)^{a}$.

\begin{tabular}{|c|c|c|}
\hline Groups & November 2016 & February 2017 \\
\hline $\mathrm{I}^{\mathrm{a}}$ & 27.78 & 47.22 \\
\hline $\mathrm{II}^{\mathrm{b}}$ & 42.86 & 71.43 \\
\hline $\mathrm{II}^{\mathrm{c}}$ & 60.87 & 86.96 \\
\hline
\end{tabular}

${ }^{*}$ Asymptomatic Plasmodium falciparum infection; a Children aged between $2-5$ years; ${ }^{b}$ Children aged between $6-9$ years; ${ }^{\circ} \mathrm{Children}$ aged between $10-13$ years.

Table 2: APFI" frequency (confirm by microscopy). 


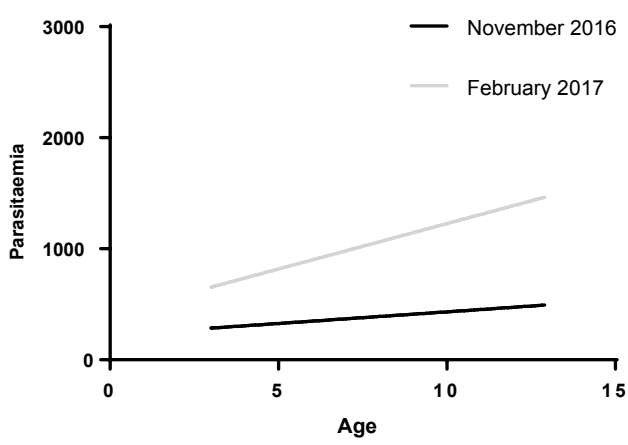

Figure 1: Correlation between parasitemia and ages.

\begin{tabular}{|c|c|c|c|}
\hline & November 2016 & February 2017 & $P$ value \\
\hline \multicolumn{4}{|c|}{ Hemoglobin level: mean \pm SD } \\
\hline$I^{a}$ & $12.56 \pm 2.13$ & $10.72 \pm 1.45$ & $<0.0001^{*}$ \\
\hline $\mathrm{II}^{\mathrm{b}}$ & $10.76 \pm 2.04$ & $10.17 \pm 1.59$ & 0.08 \\
\hline III ${ }^{\mathrm{c}}$ & $11.95 \pm 1.08$ & $10.31 \pm 1.37$ & $<0.0001^{*}$ \\
\hline \multicolumn{4}{|c|}{ Anemia: \% [mean $\mathrm{Hb}^{\mathrm{d}} \pm \mathrm{SD}$ ] } \\
\hline No anemia & $51.25(13.28 \pm 1.02)$ & $18.75(12.65 \pm 0.62)$ & $0.0040^{*}$ \\
\hline Moderate anemia $^{f}$ & $38.75(11.16 \pm 0.52)$ & $50.00(10.63 \pm 0.64)$ & $0.0001^{*}$ \\
\hline Mild anemia ${ }^{9}$ & $6.25(9.12 \pm 1.19)$ & $28.75(9.04 \pm 0.37)$ & 0.00469 \\
\hline Severe anemia ${ }^{h}$ & $3.75(5.60 \pm 1.25)$ & $2.50(6.80 \pm 0.14)$ & 0.1183 \\
\hline
\end{tabular}

*Significant $p$ value: $<0.05$; ${ }^{a}$ Children aged between $2-5$ years; ${ }^{b}$ Children aged between 6-9 years; ${ }^{\circ}$ Children aged between 10-13 years; ${ }^{d}$ Hemoglobin level; ${ }^{\mathrm{e}} \mathrm{Hb}>11.5 \mathrm{gdl} ;{ }^{\mathrm{f}} \mathrm{Hb}$ between $10-11.4 \mathrm{~g} / \mathrm{dl} ;{ }^{9} \mathrm{Hb}$ between $8-9.9 \mathrm{~g} / \mathrm{dl} ;{ }^{\mathrm{h}} \mathrm{Hb}<8 \mathrm{~g} / \mathrm{dL}$.

Table 3: Hemoglobin and Anemia profiles of APFI children.

\begin{tabular}{|c|c|c|c|}
\hline & $I^{a}$ & II $^{\mathbf{b}}$ & III' \\
\hline APFI frequency ${ }^{d}$ & 50.00 & 77.78 & 92.86 \\
\hline Anemia frequency ${ }^{e}$ & 100.00 & 100.00 & 92.31 \\
\hline
\end{tabular}

${ }^{a}$ Children aged between 2-5 years; ${ }^{b}$ Children aged between 6-9 years; ${ }^{\circ}$ Children aged between 10-13 years; d:frequency of participants with positive parasitaemia

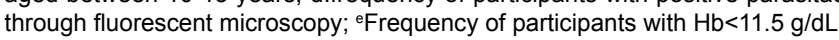

Table 4: Frequency of relapse cases.

\begin{tabular}{|c|c|c|c|}
\hline & November 2016 & February 2017 & Correlation: $r(p)^{c}$ \\
\hline APFI frequency ${ }^{a}$ & 41.25 & 65 & \multirow[t]{2}{*}{$0.93(0.002)$} \\
\hline Anemia frequency ${ }^{b}$ & 48.75 & 83.25 & \\
\hline
\end{tabular}

${ }^{a}$ Frequency of participants with positive parasitemia through fluorescent microscopy; ' ${ }^{b}$ Frequency of participants with $\mathrm{Hb}<11.5 \mathrm{~g} / \mathrm{dL}$; ' Correlation coefficient [p value].

Table 5: Correlation between APFI and associated Anemia.

23] were similarly exposed to malaria. People living in regions with intense perennial transmission might easily acquire partial immunity to malaria; thereby leading to common cases of subclinical malaria or asymptomatic carriers of malaria parasites [20,24,25]. Children from such settings probably acquire an "anti-disease immunity" rapidly which results in the reduction of mortality or severe clinical disease early on in life [25]. Thus, the persistence of APFI within this cohort suggests a state of acquired and persistent partial immunity among children living within this region. Continuous exposure to the malaria parasites is relevant for such immunity and protection to severe disease is transient this context clearly suggesting an immunity mediated as a result of the presence of the parasites in contrast solid memory immunity arising as a result on previous infections $[3,8,25]$. In addition the mechanism of protective immunity differs between meso or hypoendemic transmission zones from those occurring in hyper- to holoendemic transmission areas [24,25].
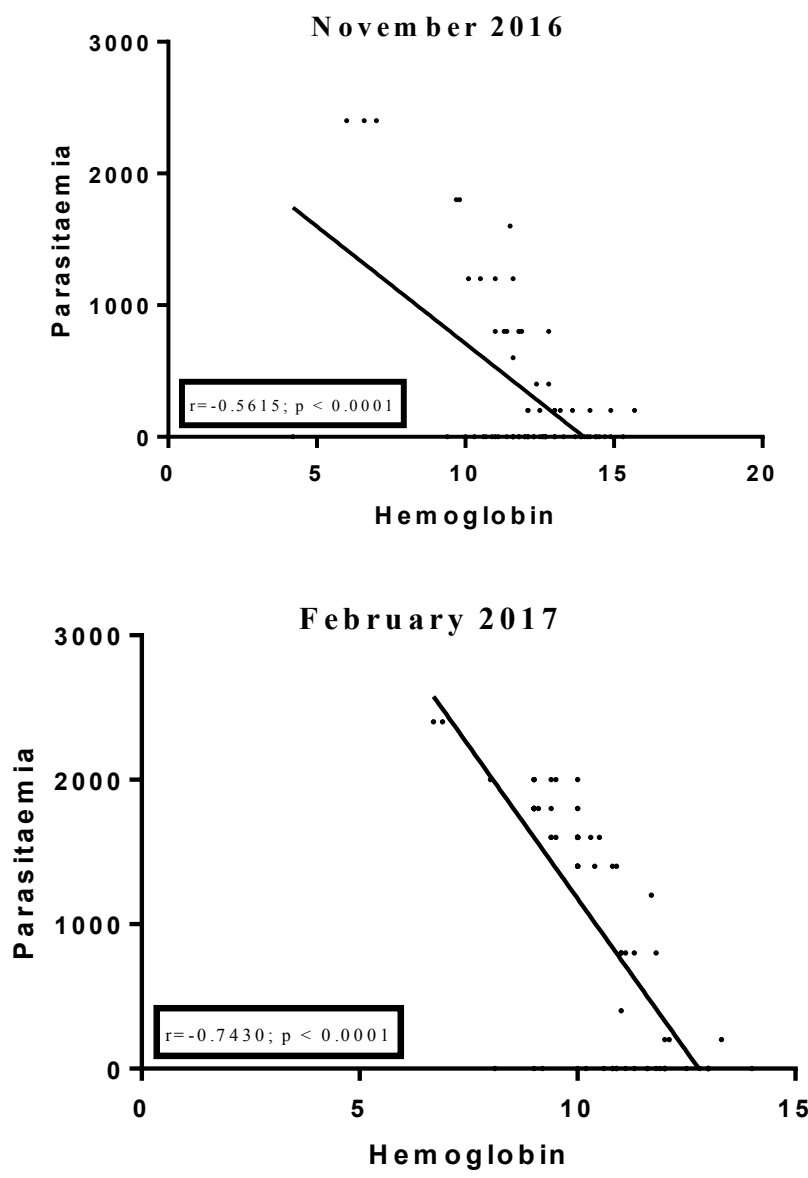

Figure 2: Correlation between Plasmodium falciparum parasitemia and hemoglobin level.

We demonstrated in this study that the rate of APFI increases with age with children older than 5 being more affected than those under 5 years old [25]. Anyway, the age of onset of the acquired immunity is somewhat earlier with heavier transmission, but protection rarely occurs before the age of 2 years; the frequency of clinical disease begins to diminish and the risk of mortality sharply decreases at about 2 to 5 years of age [25]. Similarly Gupta et al. using a mathematical modeling approach showed that immunity to noncerebral severe malaria may be acquired after only one or two infections [26] but the basis of that protection and how it fails in African children each year are very poorly understood.

Malaria infected children were treated with a three day six-dose regimen of artemether-lumefantrine (AL). This is mainly because the efficacy of the six-dose regimen of AL has been confirmed globally, even in areas of widespread parasite resistance. This six-dose AL regimen is currently the approved treatment regimen for acute, uncomplicated $P$. falciparum malaria in adults and paediatric patients with a body weight $\geq 5 \mathrm{~kg}$, irrespective of the immune status of the patients to $P$. falciparum and of the local multidrug resistance situation. This tenable in majority of countries in Africa, Asia, Europe and Latin America where the drug is registered $[27,28]$. To eliminate bias the drugs were administered without fatty food as recommended by the manufacturers. Nevertheless persistent detectable parasitaemia was observed four months after treatment in well over $50 \%$ of participants 2-5 years old. Detectable subclinical parasitaemia increased with age starting from $78 \%$ in 
participants 6-9 years old and $93 \%$ for participants $10-13$ years old. This probably indicates that the higher exposition rate leads re-infection of children, and when consider the 27-30 days needed to complete the life cycle of Plasmodium spp in human [28,29] and the half-life of the lumefantrine up to 10 days [27], it would take about two months for parasitaemia to be detected in the blood. Secondly, the persistent rate of parasitaemia could also question the efficacy of the AL combination as far as it has been demonstrated that cumulative PCR-corrected cure rates on day 28 is $100 \%$ for amodiaquine +artesunate but $97.5 \%$ for arthermether-lumefantrine [30]; Ayogu et al demonstrated that AL combination therapy reduced fever in malaria patients but failed to totally clear parasitaemia density [28]. More studies are needed to determine the impact of AL combination on the parasitaemia clearance among asymptomatic carriers of Plasmodium falciparum.

The rate of APFI in our study population increase independently of the availability of the LLIN. Less than $50 \%$ of children reported regularly using LLIN, despite their massive distribution by the Cameroon Ministry of Public Health. Although individuals who own mosquito nets must use them in order to reduce the rate of the malaria transmission between dusk and dawn, to own a LLIN do not necessary mean to use it as far as people may use it for other purposes. This is the case as reported for some villages in the North-West region of Cameroon, where $18.2 \%$ of a population used their LLINs for other purposes, such as fishing, nursing seeds and footfall nets [31]. Standing water, environmental factors including presence of grasses, outdoor activities between dusk and dawn are important risk factors for malaria transmission [32]. More efforts are therefore needed especially in these remote areas to improve the distribution of LLIN, and also to ensure the proper use of LLINs by the population in malaria hyperendemic areas.

We observed that persistent APFI correlated positively with increasing anemia, suggesting that a persistent APFI may drive anemia. In the African continent, where Plasmodium falciparum is the most prevalent human malaria parasite, anemia is responsible for about half of the malaria-related deaths [33] and specific haematological changes associated with malaria infection may vary with the level of malaria endemicity [34], malaria immunity [35] and the parasite species. Akiyana et al in 2016 demonstrated that asymptomatic malaria was associated with anemia in children of the Lao People's Democratic Republic [19]. Anemia among children has been known as one of the major consequences of subclinical malaria and is common among children heavily exposed to the malaria parasite. In addition the greatest disease risk for these children is severe anemia rather than cerebral malaria or failure of respiratory, renal, or hepatic systems $[25,26]$. Central Africa, West Africa and south Asia have the lowest mean concentrations of hemoglobin and the highest prevalence of anemia in children as reported in 2011 [36]. Similarly most African countries with lowest mean concentrations of hemoglobin and the highest anemia prevalence in children have the highest rate of $P$. falciparum infection [36,37]. So the asymptomatic character of Plasmodium falciparum parasitaemia in children do not exclude any risk of anemia; it suggest that haemolysis of red blood cells, increased clearance of infected and uninfected cells and complement deposition on the surface of red blood cells occurs and induce anemia among APFI children [37,38]. Furthermore, while during acute malaria infection there is a rapid drop in hemoglobin, during chronic malaria infection there is a slower decline; this appear to be due to increased extravascular haemolysis of red blood cells with a concomitant failure of the bone marrow to increase red cell production to compensate for these losses [37-39].

\section{Conclusion}

Persistent APFI in a remote area of Cameroon correlated positively with moderate anemia. Thus innocuous asymptomatic Plasmodium falciparum parasitaemia might be adriving force behind persistent anemia in school age children in rural rainforest areas of Cameroon. The findings provide a baseline for evidence-based planning and implementation of malaria control activities within these areas.

\section{Ethics Approval and Consent to Participate}

This study received ethical approval from the Cameroon National Ethics Committee for Human Health Research (Reference numbers 2015/03/561/CE/CNERSH/SP and 2018/01969/CE/CNERSH/SP) and the CIRCB institutional review board (protocol number 14-11). All participants provided written informed consent. Data were processed using specific identifiers for privacy and confidentiality purposes. Clinical data generated during the course of this study was provided free of charge to all participants.

\section{Availability of Data and Materials}

All data are fully available without restriction. Data are available from the CIRCB Institutional Data Access/Ethics Committee for researchers who meet the criteria for access to confidential data. All request for Data should be addressed to the General Manager of CIRCB reachable by the following address:

Prof. Alexis Ndjolo, Director General CIRCB, BP 3077 Messa Yaounde Cameroon, Tel. +237222315450, Fax. +237222315456, email:andjolo@yahoo.com or andjolo@circb-cm.

\section{Competing Interests}

The authors have declared that no competing interests exist.

\section{Funding}

This project was funded by grants from CIRCB, EDCTP (grant \#TA.2010.40200.016) TWAS(\#12059RG/bio/af/ac G) and Canada grand challenge (\#0121-01); to Godwin W Nchinda; from Korea-Africa cooperation grant (NRF-2013K1A3A1A09076155) from the National Research Foundation of Korea funded by the Ministry of Science, ICT and Future Planning in the Republic of Korea to Chae Gyu Park; and then from the Center for NanoBiotechnology Research (CNBR) of ASU for grant \# NSF-CREST (HRD-241701) and grant \# NSF-AGEP (1432991 BKR) of National Science Foundation to Alain Bopda Waffo.

\section{Author Contributions}

Conceived and designed the experiments: G.W.N and A.B.W. Performed the experiments: O.F.H, L.N.N, A.L., A. A.N, A.S.O., P.E.A, D.T., R.G,.A.G, E.N.,A.B.W Technical assistance: M.I.O, C.G.P, L.N.N, G.O.C, E.A.A, W.N.M., L.K., J.N.C.A, C.O.E. Analyzed the data: G.W.N, O.F.H. Wrote the paper: O.F.H, G.W.N.

\section{Acknowledgement}

We thank all volunteers who consented to participate in this study. We would like to thank the personnel of the Bikop Catholic health Center and School for their help in collecting the blood samples.

\section{References}

1. World malaria report (2017) Geneva: World Health Organization. Licence: CC BY-NC-SA 3.0 IGO.

2. World Health Organization (2015) Global Technical Strategy for Malaria 20162030.

3. Chen I, Clarke SE, Gosling R, Hamainza B, Killeen G, et al. (2016) "Asymptomatic" Malaria: A Chronic and Debilitating Infection That Should Be Treated. PLoS Med 13: e1001942.

4. Cowman AF, Healer J, Marapana D, Marsh K (2016) Malaria: Biology and Disease. Cell 167: 610-624.

5. Fontenille D, Simard F (2004) Unraveling complexities in human malaria 
Citation: Ouambo HF, Ngu LN, Lissom A, Ngoh AA, Okoli AS, et al. (2019) Persistent Asymptomatic Plasmodium falciparum Driven Anemia in a Treatment Re-infection Pediatric Cohort in a Rural Health District of Cameroon. J Blood Lymph 9: 248.

transmission dynamics in Africa through a comprehensive knowledge of vector populations. Comp Immunol Microbiol Infect Dis 27: 357-375.

6. Mvondo JL (1998) Malaria in Cameroon. Training Reports for TWF investigators.

7. Malaria Operational Plan FY (2017) President's Malaria Initiative Cameroon.

8. Chourasia MK, Raghavendra K, Bhatt RM, Swain DK, Valecha N, et al. (2017) Burden of asymptomatic malaria among a tribal population in a forested village of central India: a hidden challenge for malaria control in India. Public health 147: 92-97.

9. https://planificateur.a-contresens.net/afrique/cameroun/centre/bikop/2234147. $\mathrm{html}$

10. cvuc.cm/national/index.php/fr/carte-communale

11. WHO Prequalification of Diagnostics Programme. PUBLIC REPORT. Product SD Bioline Malaria Ag P.f and SD BIOLINE Malaria Ag P.f POCT. PQDx Reference Number: PQDx 0031-012-01.

12. Kashosi1 TM, Mutuga JM, Byadunia DS, Mutendela JK, Mulenda B, et al. (2017) Performance of SD Bioline Malaria Ag Pf/Pan rapid test in the diagnosis of malaria in South-Kivu, DR Congo. Pan Afr Med J 27: 216.

13. llombe G, Maketa V, Mavoko HM, da Luz RI, Lutumba P, et al. (2014) Performance of HRP2-based rapid test in children attending the health centre compared to asymptomatic children in the community. Malaria Journal 13: 308.

14. Malaria Diagnostics Technology Landscape: Enhanced Visual Parasite Detection. Project DIAMETER (Diagnostics for Malaria Elimination Toward Eradication).

15. Lifetechnologies.com/EVOS

16. http://www.sysmex-partec.com/applications/essential/malaria.html

17. Doolan DL, Dobano C, Baird JK (2009) Acquired immunity to malaria. Clin Microbiol Rev 22: 13-36.

18. Worku L, Damtie D, Endris M, Getie S, Aemero M (2014) Asymptomatic Malaria and Associated Risk Factors among School Children in Sanja Town, Northwest Ethiopia. International Scholarly Research Notices 2014: 303269.

19. Akiyama T, PongvongsaT, Phrommala S, Taniguchi T, Inamine $Y$, et al. (2016) Asymptomatic malaria, growth status, and anemia among children in Lao People's Democratic Republic: a cross-sectional study. Malar J 15:499.

20. World Malaria Report (2016) Geneva: World Health Organization. Licence: CC BY-NC-SA 3.0 IGO.

21. Dalrymple U, Mappin B, Gething PW (2015) Malaria mapping: understanding the global endemicity of falciparum and vivax malaria. BMC Medicine.

22. Alelign A, Dejene T (2016) Current Status of Malaria in Ethiopia: Evaluation of the Burden, Factors for Transmission and Prevention Methods. Acta Parasitologica Globalis 7: 1-6.

23. ACTwatch Group, Phanalasy S (2017) The malaria testing and treatment landscape in the southern Lao People's Democratic Republic (PDR). Malar J 16: 169 .
24. Brady OJ, Godfray CJ, Tatem AJ, Gething PW, Cohen JM, et al. (2015) Adult vector control, mosquito ecology and malaria transmission. Int Health 7: 121129.

25. Doolan DL, Doban C, Baird JK (2009) Acquired Immunity to Malaria. Clinical microbiology reviews pp: 13-36.

26. Gupta S, Snow RW, Donnelly CA, Marsh K, Newbold C (1999) Immunity to non-cerebral severe malaria is acquired after one or two infections. Nat Med 5: 340-3.

27. Makanga M, Krudsood S (2009) The clinical efficacy of artemether/lumefantrine (Coartem). Malar J 8: S5.

28. Ayogu EE, Ukwe CV, O Nna E (2015) Therapeutic Efficacy of ArtemetherLumefantrine for Treatment of Uncomplicated Plasmodium falciparum Malaria in Enugu, Nigeria. Trop J Pharm Res 14: 1487.

29. Cowman AF, Healer J, Marapana D, Marsh K (2016) Malaria: Biology and Disease. Cell 167: 610-624.

30. Koram KA, Abuaku B, Duah N, Quashie N (2005) Comparative efficacy of antimalarial drugs including ACTs in the treatment of uncomplicated malaria among children under 5 years in Ghana. Acta Trop 95: 194-203.

31. Ntonifor $\mathrm{NH}$, Veyufambom S (2016) Assessing the effective use of mosquito nets in the prevention of malaria in some parts of Mezam division, Northwest Region Cameroon. Malar J 15: 90.

32. Mayala BK, Fahey CA, Wei D, Zinga MM, Bwana VM, et al. (2015) Knowledge perception and practices about malaria, climate change, livelihoods and food security among rural communities of central Tanzania. Infect Dis Poverty 4: 21

33. Quintero JP, Siqueira AM, Tobón A, Blair S, Moreno A, et al. (2011) Malariarelated anemia: a Latin American perspective. MemInst Oswaldo Cruz 106: 91-104.

34. Idro R, Aloyo J, Mayende L, Bitarakwate E, John CC, et al. (2006) Severe malaria in children in areas with low, moderate and high transmission intensity in Uganda. Trop Med Int Health 11: 115-124.

35. Langhorne J, Ndungu FM, Sponaas AM, Marsh K (2008) Immunity to malaria: more questions than answers. Nat Immunol 9: 725-732.

36. Stevens GA, Finucane MM, De-Regil LM, Paciorek CJ, Flaxman SR, et al (2013) Global, regional, and national trends in hemoglobin concentration and prevalence of total and severe anemia in children and pregnant and non-pregnant women for 1995-2011: a systematic analysis of populationrepresentative data. Lancet Glob Health 1: PE16-E25.

37. Bawah AT, Nyakpo KT, Ussher FA, Alidu H, Dzogbo JJ, et al. (2018) Hematological profile of children under five years with malaria at the $\mathrm{Ho}$ Municipality of Ghana. Edorium J Pediatr 2: 100004P05AB2018.

38. Kai OK, Roberts DJ (2008) The pathophysiology of malarial anemia: where have all the red cells gone? BMC Medicine 6: 24 .

39. Lamikanra AA, Brown D, Potocnik A, Casals-Pascual C, Langhorne J, et al. (2007) Malarial anemia: of mice and men. Blood 110: 18-28. 